\title{
Round-Trip Emergency Supply Distribution Model Based on Nonfixed Routes
}

\author{
Yanli Tang $\mathbb{D}$, Ying Xiong $\mathbb{D}$, and Ying Chen \\ School of Architecture, Changsha University of Science and Technology, Changsha 410076, China \\ Correspondence should be addressed to Ying Xiong; 87854789@qq.com
}

Received 28 June 2021; Revised 8 January 2022; Accepted 12 January 2022; Published 16 February 2022

Academic Editor: Alessandro Severino

Copyright (C) 2022 Yanli Tang et al. This is an open access article distributed under the Creative Commons Attribution License, which permits unrestricted use, distribution, and reproduction in any medium, provided the original work is properly cited.

\begin{abstract}
In the face of increasing natural or man-made disasters, rapid and effective emergency dispatch and organization are of great significance to ensure the life safety of people and reduce social losses. In view of the long duration, strong demand urgency, and relatively limited transportation capacity after catastrophic events, this paper proposes a round-trip emergency supply distribution model based on nonfixed routes. This model includes two main features: (1) round trip: emergency vehicles can travel back and forth to distribute supplies; (2) unfixed routes: distribution routes of the same emergency vehicle could be variable in different trips. In order to ensure the timeliness and fairness of the supply distribution scheme, the model objective function is set to minimize the total supplies' waiting time at all demand points. According to model features, 4 constraints are set, including flow balance, capacity, vehicle scheduling, and time window. On this basis, a compound algorithm combining 2-opt and tabu search is designed to obtain the optimal plan of the model. To verify the effectiveness and superiority of the model and solution method, a case study based on the Sioux Falls network is carried out. Compared with the traditional method, the objective function is optimized by $11.92 \%$. In fact, under the control of multiple constraint conditions, the model well fits the actual application scenarios, which can provide theoretical guidance and decision support for the distribution of relevant emergency supplies.
\end{abstract}

\section{Introduction}

As the human society continues to develop, natural or manmade catastrophic events have become increasingly frequent. In the face of these events, rapid and effective emergency rescue is of great significance for reducing disaster damage and ensuring the safety of people's lives. Supply distribution is the basic premise of emergency rescue, which includes emergency supply allocation and route planning [1]. In fact, scientific and reasonable distribution of emergency supplies can improve the effectiveness of emergency rescue, thereby achieving social benefits.

In recent years, the emergency supply distribution problem has attracted more and more attention and been widely studied. In terms of research object, it is a typical vehicle routing planning problem (VRP). Most previous studies assume that emergency vehicle transportation is a one-way trip. In other words, emergency vehicles deliver supplies from the distribution centers to demand points and will not return to the distribution centers after completing their tasks. In addition, each emergency vehicle only serves one demand point, significantly reducing the complexity of the model. Based on the above assumptions, Li et al. [2] established a supply distribution model considering the timeliness and fairness and proposed a hybrid genetic algorithm to solve this problem. Xing et al. [3] constructed a comprehensive emergency scheduling model. They obtained the resource allocation plan based on multiobjective path planning. Meanwhile, Wei et al. [4] introduced the constraint conditions of resources and time windows into their model construction to improve the practicability of the model, which can output emergency supply allocation and vehicle routing plans at the same time. Based on the same one-way travel assumption, Xiong et al. [5] constructed a multimodal emergency supply distribution model integrating vehicles and helicopters. In addition, Yingli [6] 
added a penalty function based on time delay to the emergency supply distribution model to achieve a balanced distribution of supplies between demand points.

With the assumption of one-way trip, the model does not need to consider the return of vehicles, which could help simplify the model effectively, but the assumption is not completely consistent with the actual situation [7]. Because the total number of emergency vehicles is limited, it is difficult to complete all supply distribution at one time based on the assumption of one-way trip. Therefore, in most cases, emergency vehicles need to travel back and forth between distribution centers and disaster sites many times to distribute supplies gradually. Therefore, some studies proposed a roundtrip distribution plan or a multipoint distribution plan, where an emergency vehicle can serve multiple demand points in the same trip. Jun et al. [8] studied the emergency supply distribution problem with a single depot and fuzzy demand. They constructed a dynamic scheduling model and designed a particle swarm optimization algorithm to solve this problem. Based on the multitraveling salesman problem, Ming and Peiyong [9] aimed to minimize the total travel cost of the vehicles, conducting emergency supply scheduling. Zhuo et al. [10] considered the mixed transportation of self-owned and rented vehicles in the distribution center and constructed a multiobjective emergency supply distribution model. Chen et al. [11] introduced the reliability of supplies as a factor into the emergency logistics network to solve the emergency supply distribution problem under risk propagation. Furthermore, considering that, in the postdisaster rescue, the number of supplies and transportation capacity of each rescue site may be unbalanced, Han et al. [12] set the supply transfer site in their model to effectively transfer supplies.

In this study, a round-trip emergency supply distribution model based on nonfixed routes is proposed. The main features of the model include round trips, where emergency vehicles can travel back and forth to distribute supplies, and nonfixed routes, where different from the traditional method, the emergency vehicle can deliver supplies to multiple demand points in the same trip, and its distribution routes may vary in different trips. By adding these two features, the rationality and efficiency of the obtained supply distribution plan can be improved. In addition, four types of constraint conditions are set, that is, flow balance, capacity, vehicle scheduling, and time window. In order to solve the model, a compound algorithm based on 2-opt and tabu search is designed to obtain the optimal emergency supply distribution plan of the model.

\section{Model Formulation}

\subsection{Hypotheses}

(1) Through the information collection before emergency dispatching, the demands of supplies of each demand point and the supply reserves of each distribution center can be determined as known conditions. In addition, the total supply reserves should exceed the total demand of supplies of all demand points.

(2) According to specific conditions, different methods can be used to obtain the travel time of emergency vehicles. If there are emergency lanes on the road section, it could be considered that the emergency vehicles travel at a free-flow speed; otherwise, the travel time of the emergency vehicle should be obtained according to the function of the Bureau of Public Roads (BPR) [13].

$$
t_{(i, j)}= \begin{cases}t_{(i, j)}^{0}, & \text { if the bus lane is set, } \\ t_{(i, j)}^{0}\left[1+\beta\left(\frac{C_{t}}{C_{(i, j)}}\right)^{\alpha}\right], & \text { otherwise, }\end{cases}
$$

where $t_{(i, j)}$ represents the actual travel time of the road section $(i, j), \mathrm{t}_{(\mathrm{i}, \mathrm{j})}^{0}$ represents the travel time under free flow, $C_{t}$ represents the traffic volume, $C_{(i \text {, }}$ j) represents the normal road capacity, and $\alpha$ and $\beta$ represent the parameters $(\alpha=4$ and $\beta=0.15)$.

(3) The travel of emergency vehicles from the distribution center to the demand points and back to the distribution center is defined as a trip. As shown in Figure 1, during each trip, the emergency vehicle departs from the corresponding distribution center with a full load and returns to the distribution center after completing the supply distribution.

2.2. Objective Function. The objective function of the model is set to minimize the total waiting time at all demand points to ensure the timeliness and fairness of the supply distribution plan.

$$
\begin{aligned}
\operatorname{Min}\left(T_{\text {wait }}\right) & =\sum_{v \in V} \sum_{k=1}^{K} \sum_{i \in E} \theta_{v i}^{k} t_{v i}^{k} q_{v i}^{k}, \\
t_{v i}^{k} & =\sum_{n=1}^{k} \sum_{(x, y) \in A_{v i}^{k}} \delta_{v x y}^{n} T_{x y}+\sum_{n=1}^{K} \sum_{z \in N_{v i}^{k}} \theta_{v z}^{n} d .
\end{aligned}
$$

Equation (2) is to obtain the total waiting time for supplies of all demand points, that is, the product sums of waiting time and waiting quantity of supplies at all demand points. Equation (3) is used to calculate the time for emergency vehicles to arrive at the demand point in each trip, that is, the waiting time for supplies at the demand point.

\subsection{Constraint Conditions. (1) Flow balance constraint:}

$$
\sum_{i \in N} \delta_{v i h}^{k}=\sum_{j \in N} \delta_{v h j}^{k}, h \in N, \forall v \in V, k \leq K
$$




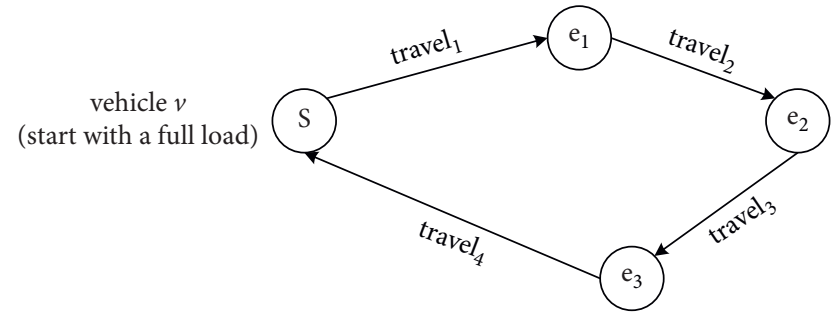

Figure 1: A trip of an emergency vehicle $v$.

(2) Capacity constraint:

$$
\begin{aligned}
& m \cdot C A P_{v} \leq \sum_{i \in E} \theta_{v i}^{k} q_{v i}^{k} \leq C A P_{v}, \forall v \in V, k \leq K, \\
& \sum_{v \in V} \sum_{k \in 1}^{k} \theta_{v i}^{k} q_{v i}^{k}=D E M_{i}, \forall i \in E, \\
& \sum_{v \in V} \sum_{k=1}^{K} \sum_{i \in E} q_{v i}^{k} \leq \sum_{j \in S} Q_{j} .
\end{aligned}
$$

(3) Vehicle scheduling constraint:

$$
\begin{aligned}
& \sum_{v \in V} \sum_{k=0}^{K} \theta_{v i}^{k} \geq 1, \forall i \in E, \\
& \sum_{v \in V} \sum_{i \in E} \delta_{v j i}^{k} \leq N_{V}, \forall j \in S, k \leq K, \\
& K-1 \leq \sum_{k}^{K} \sum_{i \in E} \delta_{v j i}^{k} \leq K, \quad \forall j \in S, v \in V .
\end{aligned}
$$

(4) Time window constraint:

$$
\delta_{v i j}^{k}\left(t_{v i}^{k}+d+T_{i j}\right) \leq t_{v j}^{k} \leq T_{i}, \forall i, j \in N, v \in V, k \in K .
$$

The four constraint conditions of the model are the flow balance, capacity, vehicle scheduling, and time window. Constraint (4) ensures the flow balance, that is, for any distribution center or demand point, the inflow and outflow of vehicles must be equal. Constraint (5) indicates that emergency vehicles can serve different demand points in the same trip. Constraint (6) requires that the demands of supplies of all demand points in the network be met. Constraint (7) ensures that the total demands of supplies at all demand points should not exceed the total reserve of supplies of all distribution centers. Constraint (8) indicates that emergency vehicles pass through all demand points and distribute supplies. Constraint (9) ensures that the number of scheduled vehicles cannot exceed the number of emergency vehicles available. Constraint (10) indicates that the emergency vehicles will not stop delivering supplies before completing the supply distribution, which limits the number of trips of the vehicle. At the same time, constraint (11) ensures that emergency vehicles must arrive at demand points on time or in advance.

\section{Solution Algorithms}

The round-trip emergency supply distribution model based on nonfixed routes is a capacitated vehicle routing problem with time window (CVRPTW), which is a typical nondeterministic polynomial hard problem $[14,15]$. Generally speaking, it is difficult to obtain an accurate and optimal solution for such problems in a short time. In fact, it is more important to quickly obtain a scientific, reasonable, and feasible solution. Therefore, a compound algorithm based on 2 -opt and tabu search is designed to solve the model $[16,17]$.

First of all, under the constraints of the time window, a preliminary supply distribution plan can be constructed using the greedy insertion algorithm. Then, a 2-opt search is performed for emergency vehicle trips serving multiple demand points to optimize the distribution order of different demand points. Following this, based on the tabu search algorithm, the supply distribution plan is globally optimized through cross-search before the optimal emergency supply distribution plan of the model can be output. The algorithm flowchart and the simplified algorithm flow table are shown in Figure 2 and Table 1.

Step 0. According to the actual situation after disaster, construct the emergency supply distribution network. Initialize, and import model parameters, such as set of distribution centers $S$, set of disaster sites $E$, and set of available emergency vehicles $V$.

Step 1. Based on the time window sequence of each disaster site, insert all disaster sites into the trips of emergency vehicles by the insertion algorithm. In each trip, the emergency vehicle starts from the distribution center, goes to disaster sites in turn to distribute supplies, and finally returns to the distribution center. Form the initial supply distribution scheme in this way, and calculate the objective function $T_{0}$. Update the initial supply distribution scheme as the current optimal solution, and set $T_{\text {wait }}=T_{0}$.

Step 2. Perform the intratrip search with 2-opt. According to the current optimal solution, update the set of all trips $R$. Select a trip passing by more than one disaster site from $R$ and operate 2-opt on it. Specifically, within the time window constraints, exchange any two disaster sites on the trip, and reverse the direction of routes between these two disaster sites. After each feasible exchange, calculate the current model objective function $T$. If $T<T_{\text {wait, }}$ accept this exchange, update the current optimal solution, and set $T_{\text {wait }}=T$; otherwise, refuse this exchange. In this way, operate 2-opt on all trips passing by more than one disaster site in $R$.

Step 3. Take the current optimal solution into the tabu list. Set the tabu length $L$, the maximum number of iterations $n_{\max }, l=0$, and $n=0$. 


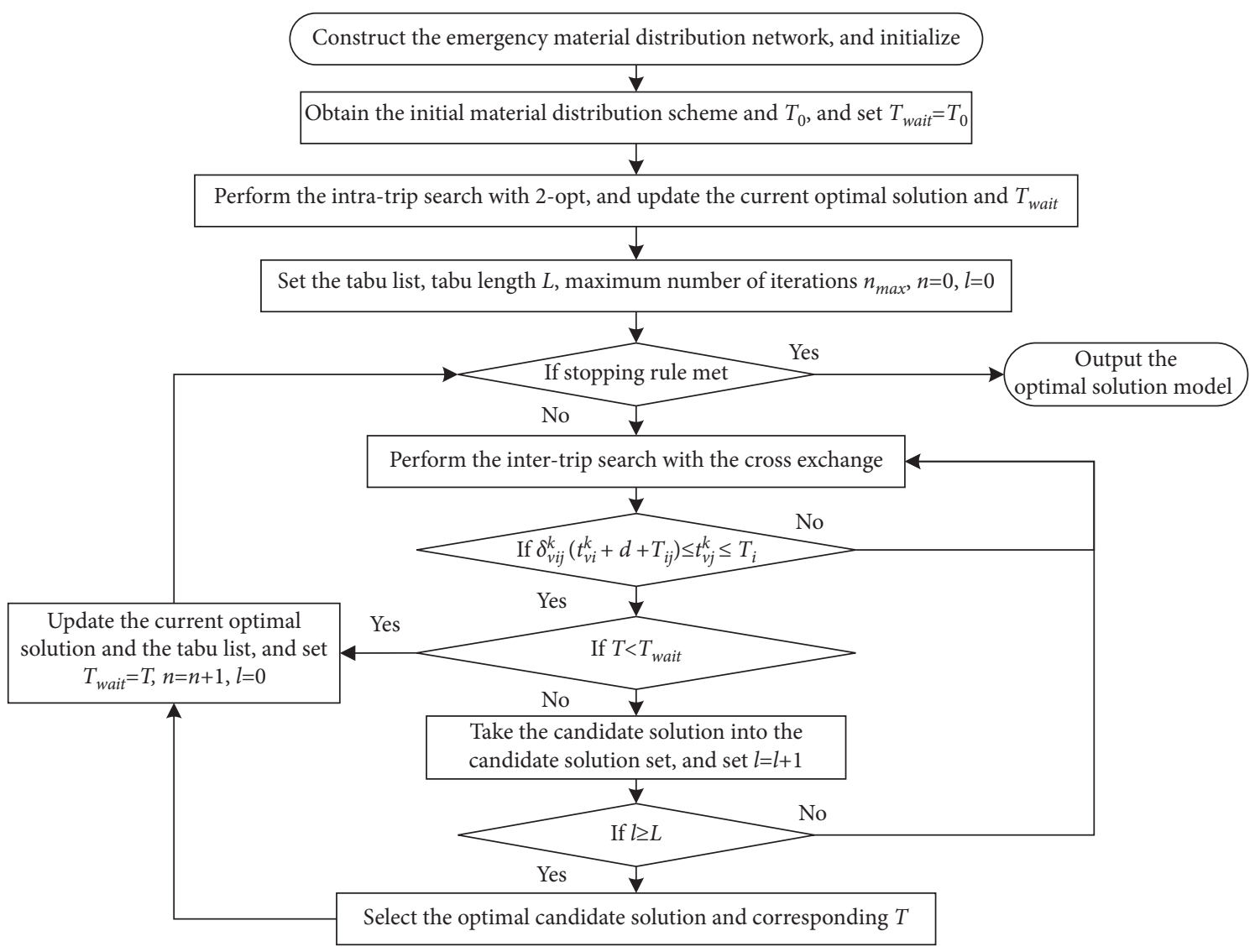

Figure 2: Algorithm flowchart.

TABLe 1: Simplified algorithm flow table.

\begin{tabular}{lc} 
Step 0 & Initialize and import model parameters \\
Step 1 & Form the initial solution \\
Step 2 & Optimize the solution with 2-opt \\
Step 3 & Take the current optimal solution into the tabu list \\
Step 4 & Form candidate solutions with the cross-exchange \\
Step 5 & Check the time window constraint \\
Step 6 & Calculate the objective function \\
Step 7 & Check the tabu length \\
Step 8 & Check the current optimal solution \\
Stopping rule & If $n \geq n_{\max }$, terminate the algorithm \\
\hline
\end{tabular}

Step 4. Perform the intertrip search with the cross-exchange. Update the candidate solution set $C$ and the set of all trips $R$. Randomly, select two trips ( $a$ and $b$ ) from $R$ and exchange the supply distribution tasks in trips $a$ and $b$ (remain the starting and ending points unchanged, and exchange the disaster sites and supply distribution). Likewise, search all the same trips as $a$ and $b$, and perform the exchange operation in pairs. In this way, generate the candidate solution.

Step 5. Based on equation (7), check the time window constraint of each disaster site. If $\delta_{v i j}^{k}\left(t_{v i}^{k}+d+T_{i j}\right) \leq t_{v j}^{k} \leq$ $T_{i}, \forall i, j \in N, v \in V, k \in K$, go to Step 6; otherwise, go to Step 4 .

Step 6. Calculate the objective function of the candidate solution $T$, and check the aspiration criterion. If $T<T_{\text {wait }}$, replace the current optimal solution with the candidate solution, update the tabu list, set $T_{\text {wait }}=T, n=n+1$, and $l=0$, and go to Step 4; otherwise, take the candidate solution into the candidate solution set $C$, set $l=l+1$, and go to Step 7 .

Step 7. Check the tabu length. If $l \geq L$, go to Step 8; otherwise, go to Step 4.

Step 8. Based on the model objective function, select the optimal candidate solution in the candidate solution set $C$, and record the corresponding objective function $T$. Replace the current optimal solution with the optimal candidate solution, update the tabu list, set $T_{\text {wait }}=T, n=n+1$, and $l=0$, and go to Step 4.

Stopping ruleIf $n \geq n_{\max }$, terminate the algorithm. Output the current optimal solution as the model optimal emergency supply distribution scheme.

\section{Case Study}

In order to verify the effectiveness of the proposed model and algorithm, a case study based on the Sioux Falls network is employed [18]. According to the location of demand points, the network topology has been processed. As shown in Figure 3, the positions and adjacent distances of all demand points in the network are determined. The setting of an emergency distribution center is generally related to storage capacity, facility conditions, 


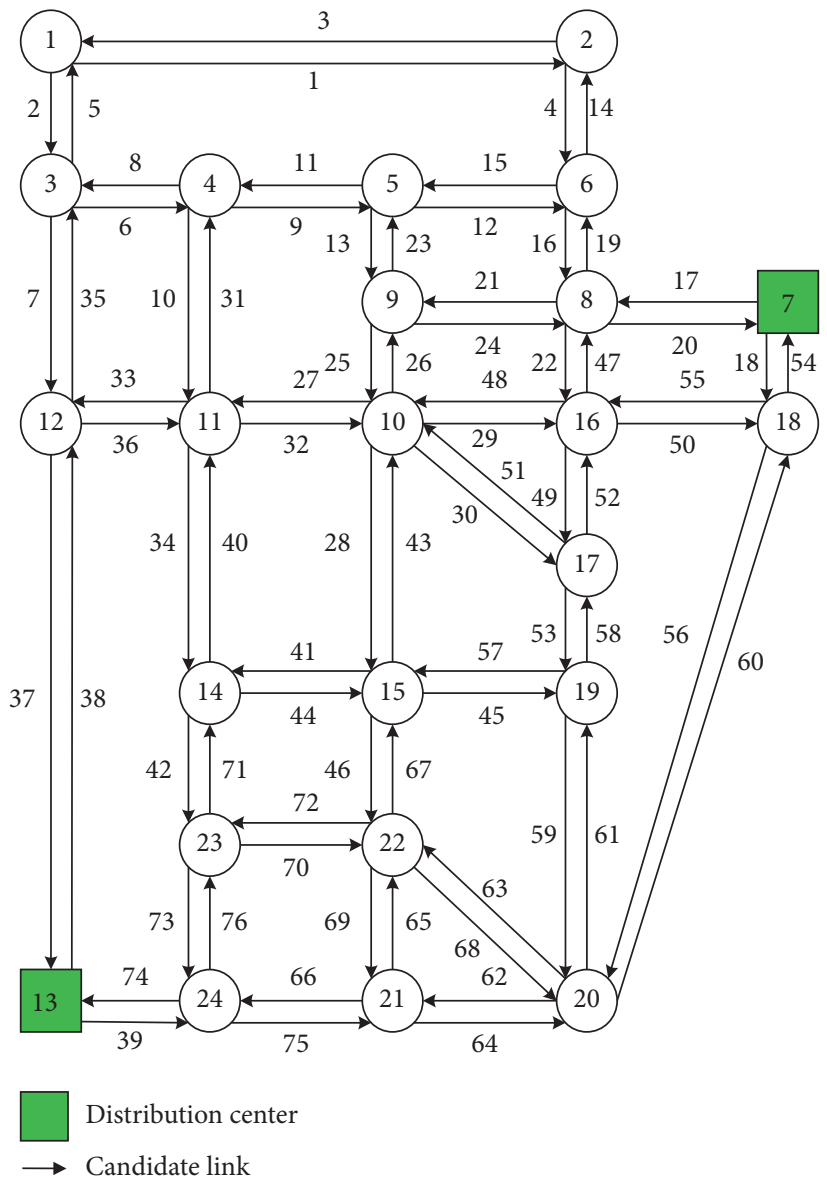

Figure 3: Sioux Falls network.

TABLE 2: Network information.

\begin{tabular}{|c|c|c|c|c|c|c|c|}
\hline Link & Time cost $(\mathrm{min})$ & Link & Time cost $(\mathrm{min})$ & Link & Time cost $(\mathrm{min})$ & Link & Time cost $(\mathrm{min})$ \\
\hline 1 & 22 & 20 & 8 & 39 & 6 & 58 & 4 \\
\hline 2 & 6 & 21 & 8 & 40 & 10 & 59 & 12 \\
\hline 3 & 22 & 22 & 4 & 41 & 8 & 60 & 24 \\
\hline 4 & 6 & 23 & 4 & 42 & 6 & 61 & 12 \\
\hline 5 & 6 & 24 & 8 & 43 & 10 & 62 & 8 \\
\hline 6 & 6 & 25 & 4 & 44 & 8 & 63 & 10 \\
\hline 7 & 8 & 26 & 4 & 45 & 8 & 64 & 8 \\
\hline 8 & 6 & 27 & 8 & 46 & 6 & 65 & 6 \\
\hline 9 & 8 & 28 & 10 & 47 & 4 & 66 & 8 \\
\hline 10 & 8 & 29 & 8 & 48 & 8 & 67 & 6 \\
\hline 11 & 8 & 30 & 10 & 49 & 6 & 68 & 10 \\
\hline 12 & 8 & 31 & 8 & 50 & 8 & 69 & 6 \\
\hline 13 & 4 & 32 & 8 & 51 & 10 & 70 & 8 \\
\hline 14 & 6 & 33 & 6 & 52 & 6 & 71 & 6 \\
\hline 15 & 8 & 34 & 10 & 53 & 4 & 72 & 8 \\
\hline 16 & 4 & 35 & 8 & 54 & 4 & 73 & 6 \\
\hline 17 & 8 & 36 & 6 & 55 & 8 & 74 & 6 \\
\hline 18 & 4 & 37 & 22 & 56 & 24 & 75 & 8 \\
\hline 19 & 4 & 38 & 22 & 57 & 8 & 76 & 6 \\
\hline
\end{tabular}

and transportation convenience. In this case, emergency distribution centers I and II are set up at nodes 7 and 13, respectively. In addition, it is assumed that each road section is equipped with an emergency lane. The travel time of emergency vehicles on each road section is shown in Table 2.

In this case, medical masks are taken as an example to conduct emergency supply distribution. The corresponding 
TABLE 3: Optimal model of the emergency supply distribution plan.

\begin{tabular}{|c|c|c|c|c|}
\hline Emergency vehicle & Plan & Trip 1 & Trip 2 & Trip 3 \\
\hline A & $\begin{array}{c}\text { Route } \\
\text { Timetable (min) } \\
\text { Supply (case) }\end{array}$ & $\begin{array}{l}7 \longrightarrow 10 \longrightarrow 7 \\
0 \longrightarrow 22 \longrightarrow 44 \\
0 \longrightarrow 40 \longrightarrow 0\end{array}$ & 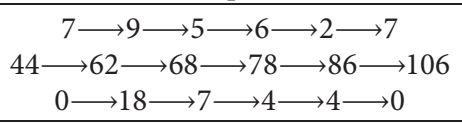 & $\begin{array}{c}7 \longrightarrow 17 \longrightarrow 19 \longrightarrow 7 \\
106 \longrightarrow 126 \longrightarrow 132 \longrightarrow 156 \\
0 \longrightarrow 26 \longrightarrow 14 \longrightarrow 0\end{array}$ \\
\hline B & $\begin{array}{c}\text { Route } \\
\text { Timetable (min) } \\
\text { Supply (case) }\end{array}$ & $\begin{array}{c}7 \longrightarrow 10 \longrightarrow 4 \longrightarrow 3 \longrightarrow 1 \longrightarrow 7 \\
0 \longrightarrow 22 \longrightarrow 40 \longrightarrow 48 \longrightarrow 56 \longrightarrow 98 \\
0 \longrightarrow 10 \longrightarrow 13 \longrightarrow 3 \longrightarrow 10 \longrightarrow 0\end{array}$ & 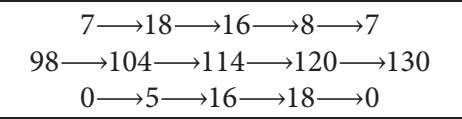 & \\
\hline $\mathrm{C}$ & $\begin{array}{c}\text { Route } \\
\text { Timetable (min) } \\
\text { Supply (case) } \\
\end{array}$ & $\begin{array}{c}13 \longrightarrow 22 \longrightarrow 15 \longrightarrow 13 \\
0 \longrightarrow 22 \longrightarrow 30 \longrightarrow 58 \\
0 \longrightarrow 17 \longrightarrow 23 \longrightarrow 0\end{array}$ & $\begin{array}{c}13 \longrightarrow 24 \longrightarrow 23 \longrightarrow 14 \longrightarrow 13 \\
58 \longrightarrow 66 \longrightarrow 74 \longrightarrow 82 \longrightarrow 102 \\
0 \longrightarrow 12 \longrightarrow 16 \longrightarrow 12 \longrightarrow 0\end{array}$ & \\
\hline $\mathrm{D}$ & $\begin{array}{c}\text { Route } \\
\text { Timetable (min) } \\
\text { Supply (case) }\end{array}$ & $\begin{array}{c}13 \longrightarrow 21 \longrightarrow 20 \longrightarrow 13 \\
0 \longrightarrow 16 \longrightarrow 26 \longrightarrow 50 \\
0 \longrightarrow 12 \longrightarrow 21 \longrightarrow 0\end{array}$ & $\begin{array}{c}13 \longrightarrow 14 \longrightarrow 13 \\
50 \longrightarrow 70 \longrightarrow 90 \\
0 \longrightarrow 30 \longrightarrow 0\end{array}$ & $\begin{array}{c}13 \longrightarrow 12 \longrightarrow 11 \longrightarrow 13 \\
90 \longrightarrow 114 \longrightarrow 122 \longrightarrow 152 \\
0 \longrightarrow 15 \longrightarrow 25 \longrightarrow 0\end{array}$ \\
\hline
\end{tabular}

TABLE 4: Subgraph division in the control solution.

\begin{tabular}{lcc}
\hline Distribution center & Service range & Demands of supply (case) \\
\hline I & $2,4,5,6,7,8,9,10,16,17,18,19$ & 188 \\
II & $1,3,11,12,13,14,15,20,21,22,23,24$ & 212 \\
\hline
\end{tabular}

TABle 5: Control plan.

\begin{tabular}{|c|c|c|c|c|}
\hline Emergency vehicle & Plan & Trip 1 & Trip 2 & Trip 3 \\
\hline \multirow{3}{*}{ A } & Route & $7 \longrightarrow 10 \longrightarrow 7$ & $7 \longrightarrow 18 \longrightarrow 17 \longrightarrow 19 \longrightarrow 7$ & $7 \longrightarrow 6 \longrightarrow 9 \longrightarrow 7$ \\
\hline & Timetable (min) & $0 \longrightarrow 22 \longrightarrow 44$ & $44 \longrightarrow 50 \longrightarrow 66 \longrightarrow 72 \longrightarrow 96$ & $96 \longrightarrow 110 \longrightarrow 124 \longrightarrow 142$ \\
\hline & Supply (case) & $0 \longrightarrow 40 \longrightarrow 0$ & $0 \longrightarrow 4 \longrightarrow 18 \longrightarrow 7 \longrightarrow 11 \longrightarrow 0$ & $0 \longrightarrow 4 \longrightarrow 18 \longrightarrow 0$ \\
\hline \multirow{3}{*}{ B } & Route & $7 \longrightarrow 16 \longrightarrow 10 \longrightarrow 4 \longrightarrow 7$ & $7 \longrightarrow 8 \longrightarrow 2 \longrightarrow 5 \longrightarrow 17 \longrightarrow 7$ & \\
\hline & Timetable (min) & $0 \longrightarrow 14 \longrightarrow 24 \longrightarrow 42 \longrightarrow 72$ & $72 \longrightarrow 82 \longrightarrow 94 \longrightarrow 110 \longrightarrow 130 \longrightarrow 150$ & \\
\hline & Supply (case) & $0 \longrightarrow 10 \longrightarrow 16 \longrightarrow 13 \longrightarrow 0$ & $0 \longrightarrow 5 \longrightarrow 15 \longrightarrow 14 \longrightarrow 0$ & \\
\hline \multirow{3}{*}{$\mathrm{C}$} & Route & $13 \longrightarrow 22 \longrightarrow 15 \longrightarrow 13$ & $13 \longrightarrow 24 \longrightarrow 23 \longrightarrow 21 \longrightarrow 13$ & $13 \longrightarrow 14 \longrightarrow 1 \longrightarrow 13$ \\
\hline & Timetable (min) & $0 \longrightarrow 22 \longrightarrow 30 \longrightarrow 58$ & $58 \longrightarrow 66 \longrightarrow 74 \longrightarrow 90 \longrightarrow 106$ & $106 \longrightarrow 126 \longrightarrow 158 \longrightarrow 196$ \\
\hline & Supply (case) & $0 \longrightarrow 17 \longrightarrow 23 \longrightarrow 0$ & $0 \longrightarrow 12 \longrightarrow 12 \longrightarrow 16 \longrightarrow 0$ & $0 \longrightarrow 23 \longrightarrow 10 \longrightarrow 0$ \\
\hline \multirow{3}{*}{$\mathrm{D}$} & Route & $13 \longrightarrow 24 \longrightarrow 12 \longrightarrow 3 \longrightarrow 13$ & $13 \longrightarrow 14 \longrightarrow 20 \longrightarrow 13$ & $13 \longrightarrow 11 \longrightarrow 13$ \\
\hline & Timetable (min) & $0 \longrightarrow 8 \longrightarrow 38 \longrightarrow 48 \longrightarrow 80$ & $80 \longrightarrow 100 \longrightarrow 126 \longrightarrow 150$ & $150 \longrightarrow 180 \longrightarrow 210$ \\
\hline & Supply (case) & $0 \longrightarrow 3 \longrightarrow 15 \longrightarrow 12 \longrightarrow 0$ & $0 \longrightarrow 21 \longrightarrow 19 \longrightarrow 0$ & $0 \longrightarrow 25 \longrightarrow 0$ \\
\hline
\end{tabular}

parameters are set as follows. Emergency distribution centers I and II reserve 200 and 250 cases of medical masks, respectively. Four emergency vehicles (A, B, C, and D) are set at I and II. The value of $m$ is set to 0.75 . The capacity of emergency vehicles is 40 cases. The loading and unloading time at the node is 2 minutes.

On this basis, the proposed model is applied to case solving. The optimal emergency supply distribution plan of the model is shown in Table 3. It can be found that the optimal solution of the model satisfies all constraint conditions, and each demand point can effectively obtain supplies within the time window, ensuring the time reliability of the plan. The total waiting time of supplies is 25,822 minutes, and the unit supply waiting time is 64.56 minutes.

In order to verify the effectiveness of the proposed model, an emergency supply distribution plan based on traditional methods is obtained as a control plan [9]. This method divides the subgraphs (the service range of each emergency distribution center) based on the multitraveling salesman problem (MTSP) and obtains the supply distribution plan in each subgraph. Under the same model constraint conditions, the obtained control plans are shown in Tables 4 and 5. The total waiting time of supplies is 29,316 minutes, and the unit supply waiting time is 73.29 minutes.

It is obvious that these two plans meet the flow balance constraint, capacity constraint, vehicle scheduling constraint, and time window constraint. However, compared with the control plan, the total supply waiting time of the optimized solution of the model is reduced by $11.92 \%$, showing better timeliness. At the same time, the total number of vehicle trips in the optimized solution of the model is reduced by 1 , indicating that its vehicle transportation utilization rate has been improved. Therefore, it can be concluded that the proposed model more effectively solves the problem of emergency supply distribution.

\section{Conclusion}

This study proposes an emergency supply distribution model, which is defined by its main features, that is, round trips and nonfixed routes. The objective function of the model is set to minimize the total waiting time of supplies of all demand points, which will help improve the timeliness and fairness of the supply distribution plan. In addition, 
according to the features of the model, the flow balance constraint, capacity constraint, vehicle scheduling constraint, and time window constraint are set, respectively. A compound algorithm based on 2-opt and tabu search is designed to solve the emergency supply distribution model. In order to verify the effectiveness of the proposed model and algorithm, a case study is carried out in the Sioux Falls network. Compared with the control plan, not only the objective function of the optimal emergency supply distribution plan of the model is reduced by $11.92 \%$ but the total number of vehicle trips is also reduced by 1 . It significantly indicates that the optimal solution of the model is more effective, which helps reduce system costs and improve rescue efficiency. Through the case study, the superiority of the proposed model and algorithm can be verified.

With the control of multiple constraint conditions, the model well fits the actual application scenarios. Compared with the traditional models, it has better applicability and practicability, which can provide theoretical guidance and decision support for the distribution of relevant emergency supplies. However, in some special cases, it may be difficult to obtain accurate supply demands of demand points the first time. Therefore, the author will further study methods to apply the model in the cases of emergency supply distribution with uncertain demands. In addition, the intelligent development of transportation is imperative. For example, smart roads, autonomous vehicles, and unmanned aerial vehicles (UAVs) have been more and more studied and applied, which own the advantages of high efficiency, convenience, and flexibility [19-21]. Hence, the author will also further explore emergency supply distribution methods to combine traditional vehicle transportation with intelligent transportation technology.

\section{Notations}

\section{Parameters}

$N: \quad$ Set of all nodes in the network

S: $\quad$ Set of distribution centers

E: $\quad$ Set of demand points

A: $\quad$ Set of arcs in the network

$V: \quad$ Set of available emergency vehicles

$N_{V}: \quad$ Number of available emergency vehicles

$D E M_{i}$ : Demand of supplies at demand point $i, i \in E$

$Q_{j}$ : $\quad$ Supply reserves of a distribution center $j, j \in S$

$T_{i j}$ : $\quad$ Time cost on $\operatorname{arc}(i, j),(i, j) \in A$

$d$ : Waiting time of emergency vehicles at nodes

$C A P v$ : Vehicle capacity of vehicle $v, v \in V$

$T_{i}: \quad$ Maximum time window before which supplies should be delivered to demand point $i, i \in E$

\section{Variables}

$\theta_{v i}^{k}:\left\{\begin{array}{l}1, \text { if vehicle } v \text { passes by disaster site } i \text { on its } k \text { th trip, } \\ 0, \text { otherwise. }\end{array}\right.$ $i \in N, v \in V, k \leq K$ $\delta_{v i j}^{k}:\left\{\begin{array}{l}1, \text { if } \operatorname{arc}(i, j) \text { is traversed by vehicle } v \text { on its } k \text { th trip, } \\ 0, \text { otherwise. }\end{array}\right.$

$(i, j) \in A, v \in V, k \leq K$

\section{Intermediate variables}

K: $\quad$ Maximum number of trips for emergency vehicles

$t_{v i}^{k}$ : Time cost measured from the departure to the arrival at node $i$ of vehicle $v$ on its $k$ th trip,

$t_{v i}^{k} \geq 0, i \in N, v \in V, k \leq K$

$q_{v i}^{k}$ : Number of supplies distributed at node $I$ by vehicle $v$ on its $k$ th trip, $q_{v i}^{k} \geq 0, i \in N, v \in V, k \leq K$

$N_{v i}^{k}$ : Set of all nodes that vehicle $v$ has passed through before arriving at node $i$ on its $k$ th trip, $i \in N, v \in V, k \leq K$

$A_{v i}^{k}$ : Set of all arcs that vehicle $v$ traverses before arriving at node $i$ on its $k$ th trip, $i \in N, v \in V, k \leq K$.

\section{Data Availability}

The data used to support the findings of this study are available from all the authors upon request and are included within the article.

\section{Conflicts of Interest}

The authors declare that they have no conflicts of interest.

\section{References}

[1] Z Yanhui, Z. H. U. Changfeng, W. Qingrong, L. Sinan, and M. Yangyang, "Bi-objective optimization of emergency material game allocation considering limited rationality," China Safety Science Journal, vol. 30, no. 11, pp. 168-174, 2020.

[2] W. Li, Z Xianchen, Z. Zhixue, and L. Liu, "Integrated Decision Making of Emergency Vehicle Allocation and Emergency Material distribution," Journal of Central South University (Science and Technology), vol. 49, no. 11, pp. 2766-2775, 2018.

[3] Z Xing, J Kang, and L. Hao, "Resource Allocation Model Based on Multi-Objective Path Planning in Emergency management," Journal of South China University of Technology (Natural Science Edition), vol. 47, no. 04, pp. 76-82, 2019.

[4] L. V. Wei, L. I. Zhihong, F. Manman, M. Dun, and Z. Xiangcheng, "Emergency material allocation in consideration of resource and time window constraints," China Safety Science Journal, vol. 29, no. 12, pp. 137-143, 2019.

[5] X. Xiong, F. Zhao, Y. Wang, and Y. Wang, "Research on the model and algorithm for multimodal distribution of emergency supplies after earthquake in the perspective of fairness," Mathematical Problems in Engineering, vol. 2019, 12 pages, 2019.

[6] W. Yingli, "Research on emergency material distribution model after fire disaster based on multivariate statistical analysis," Journal of Catastrophology, vol. 34, no. 4, pp. 53-56, 2019.

[7] L. Wei, L. Zhihong, and M. A. Yaping, "Research on route planning of emergency materials distribution vehicles considering time window of requirements by disaster point," Journal of Safety Science and Technology, vol. 16, no. 3, pp. 5-11, 2020.

[8] T. Jun, M. A. Wenzheng, W. Yingluo, and W. Kan-liang, "Emergency supplies distributing and vehicle routes 
programing based on particle swarm optimization," Systems Engineering-Theory \& Practice, vol. 31, pp. 898-906, 2011.

[9] L. Ming and Z. Peiyong, "New hybrid genetic algorithm for solving the multiple traveling salesman problem: an example of distribution of emergence materials," Journal of Systems Management, vol. 23, no. 02, pp. 247-254, 2014.

[10] L. I. Zhuo, L. I. Yinzhen, and L. I. Wenxia, "Multi-objective optimization model and solution algorithm for emergency material transportation path," Journal of Computer Applications, vol. 39, no. 09, pp. 2765-2771, 2019.

[11] T. Chen, .S WU, J. Yang, and G. Cong, "Risk propagation model and its simulation of emergency logistics network based on material reliability," International Journal of Environmental Research and Public Health, vol. 16, no. 23, p. 4677, 2019.

[12] Y. Han, Y. Yang, and Y. Peng, "Rescue vehicle routing optimization considering distribution of resources in rescue points," Natural Science Edition, Journal of Shanghai University, vol. 24, no. 4, , pp. 655-664, Shanghai,China, 2018.

[13] Z. Jie, W. Zhi-yong, and X. Wei-sheng, "Model and solution of rescue path selection in emergency," Application Research of Computers, vol. 28, no. 4, pp. 1311-1314, 2011.

[14] S. Shahparvari, B. Abbasi, and P. Chhetri, "Possibilistic scheduling routing for short-notice bushfire emergency evacuation under uncertainties: an Australian case study," Omega, vol. 72, no. oct., pp. 96-117, 2016.

[15] L. I. Yan, Y. E. Chunming, and G. Jing, "Multi-cycle emergency medical material allocation considering patient panic in epidemic environment," Journal of Safety and Environment, vol. 21, no. 4, pp. 1643-1651, 2021.

[16] L. I. Yang, F. Houming, Z. Xiaonan, and Y. Xiang, "Two-phase variable neighborhood tabu search for the capacitated vehicle routing problem with fuzzy demand," Systems EngineeringTheory \& Practice, vol. 38, no. 02, pp. 522-531, 2018.

[17] X. Zhao, K. Ji, P. Xu, and W. Qian, "A round-trip bus evacuation model with scheduling and routing planning," Transportation Research Part A Policy and Practice, vol. 137, pp. 285-300, 2020.

[18] G. Ren, Z. Huang, Y. Cheng, and W. H. K. Lam, "An integrated model for evacuation routing and traffic signal optimization with background demand uncertainty," Journal of Advanced Transportation, vol. 47, no. 1, pp. 4-27, 2013.

[19] S. Trubia, A. Severino, S. Curto, and F. Arena, "Smart roads: an overview of what future mobility will look like," Infrastructure, vol. 5, no. 12, pp. -110014, 2021.

[20] S. Trubia, S. Curto, A. Severino, F. Arena, and Y. Zuccalà, "Autonomous vehicles effects on public transport systems," in Proceedings of the International Conference Of Computational Methods In Sciences and Engineering ICCMSE, vol. 2343, no. 1, 2021.

[21] H. A. N. Bing, Q. I. N. Danyang, Z. Ping, L. Ma, and M. B. Teklu, "Modeling and performance optimization of unmanned aerial vehicle channels in urban emergency management," ISPRS international journal of GEO-information, vol. 10, no. 7, 2021. 